\title{
International neue Diagnosekriterien definiert
}

Die hochauflösende Computertomographie (HRCT) gilt als Schlüssel in der Diagnostik interstitieller Lungenerkrankungen. Für die idiopathische Lungenfibrose (IPF) gilt das im Besonderen. Teils kann auf invasive Diagnostik verzichtet werden.

In der aktualisierten Leitlinie der American Thoracic Society (ATS) sowie der European Respiratory Society (ERS) zur idiopathischen Lungenfibrose (IPF) sind die Diagnosekriterien sowie Definitionen und Klassifikationen präzisiert worden. Das hat unmittelbare Auswirkungen auf die Diagnosestellung bei Verdacht auf eine interstitielle Lungenerkrankung, einer Gruppe von mehr als 200 meist seltenen Krankheiten [1]. Bei eindeutigem Befund der HRCT ist keine histologische Bestätigung einer IPF-Diagnose vonnöten und es kann eine antifibrotische Therapie eingeleitet werden. Das unterstreiche die Bedeutung und Verantwortung der Radiologie auf diesem Feld, betont Dr. Beate Rehbock, niedergelassene Radiologin aus Berlin. Zumal etwa bei Vorliegen einer rheumatischen Grunderkrankung der Patient mit interstitieller Lungenerkrankung völlig anders behandelt werde. „Eine interstitielle Lungenerkrankung kann auch mal die Erstmanifestation einer Kollagenose sein", so der Pneumologe Prof. Dr. Dirk Koschel vom Universitätsklinikum in Dresden.

\section{Eine Frage des UIP-Musters}

Zunächst ist eine HRCT dann angezeigt, wenn ein begründeter klinischer Verdacht auf eine IPF besteht und andere mögliche Ursachen oder assoziierte Krankheiten ausgeschlossen sind. Typisch ist der über 60-jährige (Ex-) Raucher mit Belastungsdyspnoe, trockenem Husten und inspiratorischem Knisterrasseln bei der Auskultation. Die Diagnose IPF kann sowohl dann gestellt werden, wenn ein typisches, aber auch wenn ein wahrscheinliches UIP (usual interstitial pneumonia)Muster vorliegt. Die zwei weiteren UIPKategorien sind "nicht eindeutiges UIPMuster" sowie "CT-Muster unvereinbar mit UIP", letzteres steht für eine alternative Diagnose.

Ein typisches UIP-Muster liegt vor bei subpleural und basal dominant verteilten Honigwaben-Zysten mit oder ohne periphere Traktionsbronchiektasen oder Bronchiolektasen. Als wahrscheinliches UIP-Muster gilt, wenn zwar keine Honigwaben, aber Retikulationen und periphere Traktionsbronchiektasen sichtbar sind. Eine milde milchglasartige Trübung ist möglich. In über $90 \%$

der Fälle kann der HRCT-Befund eines UIP-Musters durch histopathologische Untersuchungen bestätigt werden.

Bei unklarem HRCT-Befund erfolgt eine invasive Diagnostik mit bronchoalveolärer Lavage, chirurgischer Lungenbiopsie und/oder Kryobiopsie. Auch das histologische Ergebnis wird in die vier genannten Kategorien klassifiziert. Im interdisziplinären ILD-Board, das sich in der Regel aus Pneumologen, Radiologen, Pathologen und gegebenenfalls einem Rheumatologen zusammensetzt, wird schließlich aus den Befundkombinationen heraus die Diagnose gestellt.

\section{Thomas Meißner}

\section{Literatur \\ 1. RaghuG, et al (2018) Am J Respir Crit Care Med 198:e44-68}

Wien klin Mag 2019 · 22:171 https://doi.org/10.1007/s00740-01900304-5

(C) Springer-Verlag GmbH Austria, ein Teil von Springer Nature 2019
Quelle: 100. Deutscher Röntgenkongress 29. Mai - 1. Juni 2019 in Leipzig, Lunchsymposium „Aufschlag ILD: Wie würden Sie entscheiden?" am 31.05.2019, Veranstalter: Boehringer Ingelheim

Hier steht eine Anzeige. 